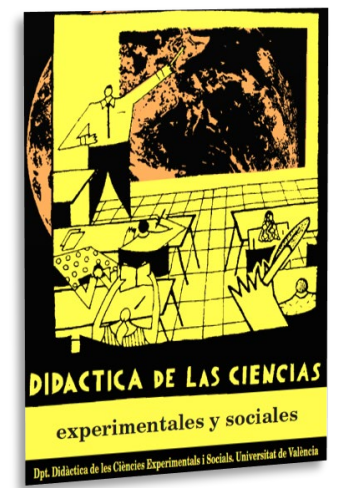

\title{
Percepciones del alumnado de Educación Secundaria (15-17 años) hacia la función social de la ciencia
}

\author{
Perceptions of Secondary Education \\ students (15-17 years old) towards the \\ social function of science
}

DOI: $10.7203 / D C E S .39 .17766$

\author{
Teresa Lupión Cobos \\ Universidad de Málaga, teluco@uma.es \\ ORCID iD: https://orcid.org/0000-0002-6937-7178 \\ Jesús Ramón Girón Gambero \\ IES Isaac Albéniz (Málaga), jesusr.giron@gmail.com \\ ORCID iD: https://orcid.org/0000-0002-6388-0178
}

\begin{abstract}
RESUMEN: En esta investigación se estudian las percepciones del alumnado de Educación Secundaria (15-17 años), en función de su género y nivel educativos (educación obligatoria y postobligatoria), hacia tres aspectos relacionados con la función social de la ciencia. Los 158 participantes cumplimentaron un cuestionario validado internacionalmente, que nos ha permitido alcanzar datos diagnósticos respecto a estos factores que indica un mayor reconocimiento sobre la importancia del papel de la Ciencia en la sociedad, frente a los restantes, que decrecen en el siguiente orden: adopción de actitudes científicas e interés respecto a ella en el tiempo de ocio, respectivamente. Finalmente se aportan implicaciones didácticas desde el campo de la Didáctica de las Ciencias Experimentales, en relación con la proyección de este estudio hacia propuestas de enseñanza futuras, que permitan poner en acción un conocimiento conectado con la identidad del estudiante, en aras al fomento de sus vocaciones científico-técnicas.
\end{abstract}

Palabras Clave: Educación Secundaria, percepciones del alumnado, implicaciones sociales de la ciencia, identidad personal, vocaciones científicas.

ABSTRACT: In this research, the perceptions of Secondary School students (15-17 years old) are studied, according to their gender and educational level (compulsory and post-compulsory education), towards three aspects related to the social function of science. The 158 participants filled in an internationally validated questionnaire, which has allowed us to obtain diagnostic data regarding these factors that indicate a greater recognition of the importance of the role of Science in society, compared to the rest, which decrease in the following order: adoption of scientific attitudes and interest towards it in leisure time, respectively. Finally, didactic implications are provided from the field of Didactics of Experimental Sciences, in relation to the projection of this study towards future teaching proposals, which would allow the implementation of a knowledge connected to the identity of the student in order to promote their scientific-technical vocations.

KEYWORDS: Secondary Education, student perceptions, social implications of science, personal identity, scientific vocations.

Fecha de recepción: junio 2020

Fecha de aceptación: diciembre 2020

Este estudio forma parte del proyecto de I+D de Excelencia "Desarrollo de competencias en problemas de la vida diaria mediante prácticas científicas de argumentación, indagación y modelización en enseñanza secundaria y universitaria (CPAIM)" (EDU2017-82197-P) financiado por el Ministerio de Ciencia, Innovación y Universidades 2017. 


\section{INTRODUCCIÓN}

En el momento educativo presente, la falta de interés hacia la Ciencia y la Tecnología que desde finales del siglo XX parece reflejar la decreciente proporción de estudiantes que cursan carreras asociadas al campo científico-tecnológico, asociado al elevado porcentaje de fracaso escolar en materias de este campo, ponen en valor la importancia de promover el interés por la cultura científica.

En la Conferencia Mundial sobre la Ciencia para el siglo XXI, auspiciada por la UNESCO y el Consejo Internacional para la Ciencia, ya se planteaba la enseñanza de las ciencias y la tecnología (CyT) como un imperativo estratégico (Declaración de Budapest, 1999), a su vez asumido como objetivo para el año 2010 (Consejo de Europa, 2003), abogando por reducir el desequilibrio en la representación de hombres y mujeres en la participación en estos campos. Iniciativa, que se convierte así en una prioridad institucional desde las administraciones educativas europeas, intentando promover sociedades competentes, reconociendo el valor decisivo de las vocaciones científicas para el futuro de los países (European Comission, 2005; 2015).

Desde el enfoque de una educación concebida como experiencia global, a lo largo de toda la vida (Delors, 1996), cambiar la realidad de este fracaso de la educación científica y movilizar cambios para su mejora, constituye una demanda y un reto para ayudar a construir una sociedad más equitativa e igualitaria, posibilitando una ciencia para todos (Fensham, 2002), que aporte el poder del conocimiento científico a la mayoría de los ciudadanos, de manera que se favorezca el progreso de todas las personas (Mayor Zaragoza, 2000). Sin embargo, este proceso es complejo y la investigación en diferentes áreas, evidencia que en las claves para el fomento de las vocaciones científico-técnicas, hay una gran influencia de la percepción que cada individuo tiene de su propia identidad, tanto personal como en relación a su entorno familiar, educativo y social (Dewitt et al., 2013; Simarro, 2015).

Tener en cuenta estos aspectos se constituye en una necesidad, requerida desde la sociedad, para facilitar una integración activa de la función social de la ciencia que ésta precisa. Así, en concreto, en la sociedad española, la valoración global que realizan de la CyT sus ciudadanos, ofrece un claro balance global positivo, a lo largo de la última década (FECYT, 2013, 2019), donde la mayoría de la población considera que los beneficios de la CyT son mayores que sus perjuicios, con porcentajes que muestran, la importancia de la relación directa de la variable nivel de formación científica y tecnológica y la confianza en la ciencia y la tecnología. Es decir, a mayor nivel de formación científica se perciben aún más las ventajas que los perjuicios de la ciencia y el reconocimiento del papel importante de la cultura científica. Asimismo, la evolución que se registra, sobre el reconocimiento social de las profesiones más asociadas a la $\mathrm{CyT}$, nos indica un prestigio cada vez mayor de éstas.

Con esta orientación es importante conocer la evolución que muestran nuestros estudiantes ante determinados factores, que pueden considerarse predictores de futuras vocaciones científicas, como: acercar la imagen de la ciencia a la sociedad, haciéndola más cercana y útil al estudiante, integrándola en su vida ciudadana; facilitar la accesibilidad hacia los estudios de carreras científicas, mostrándolos atractivos, asequibles y útiles en el desarrollo personal y social de los estudiantes, o, valorar la buena imagen social de profesiones relacionadas con la $\mathrm{CyT}$, fomentando y promoviendo futuros desempeños laborales en nuestros jóvenes.

Para ello, facilitar la reflexión y discusión acerca de las prácticas educativas cotidianas en el aula y la construcción de nuevas maneras de enseñar ciencias con las que favorecer una adecuada formación científica del alumnado, es un camino que es necesario recorrer, en el que podamos analizar las visiones deformadas de la CyT que puedan haberse transmitido por la propia enseñanza, y que están contribuyendo en estos jóvenes ciudadanos, a actitudes de rechazo o de escasa motivación hacia ellas, conformando su identidad personal y social, condicionando así decisiones futuras para su integración en la vida ciudadana. 


\section{LAS PRÁCTICAS CIENTÍFICAS COMO ESTRATEGIA DE REFLEXIÓN PARA EL ALUMNADO HACIA SU PERCEPCIÓN EN TORNO A LA FUNCIÓN SOCIAL DE LA CIENCIA}

La enseñanza tradicional de las ciencias en el aula no suele proporcionar demasiadas ocasiones al alumnado para familiarizarse con la naturaleza de la ciencia, las estrategias y características del trabajo científico y las relaciones de la ciencia respecto a la sociedad en la que se producen.

Un posible escenario para este acercamiento surge abordando un reenfoque educativo que contemple el modelo del aprendizaje de las ciencias, en cuanto a su diseño y finalidades y, a su tratamiento en el aula.

En esta senda, el marco educativo del aprendizaje permanente a lo largo de la vida (PISA, 2015) (OCDE, 2016) y las propuestas desde la educación científica, a favor de una alfabetización científica y tecnológica para todos los ciudadanos (Klahr, Immerman y Jirout, 2011; Zoller, 2012 ) conducen a escenarios que desde una formación integral de los estudiantes, partan de sus puntos de partida y valores, con un conocimiento en la acción, ante situaciones cercanas en las que el estudiante participe activamente y juegue un rol protagonista.

Desde este enfoque educativo, en nuestro país, el informe ENCIENDE (COSCE, 2011), plantea esta alfabetización en el marco de desarrollar las competencias científicas en todos los ciudadanos, promoviendo desde la educación científica, el interés y motivación hacia la ciencia y el conocimiento de las soluciones que ésta aporta en sus vidas, mediante el desarrollo de situaciones de enseñanza innovadoras (Mellado, 2011), en las que los estudiantes pongan en acción sus capacidades científicas, en torno al razonamiento, utilización de evidencias o argumentación de éstas, ante fenómenos y situaciones (Ryder, 2001; Gormally, Brickman y Lutz, 2012; Solbes et al., 2012), facilitando así que sea capaz de aplicar sus conocimientos teóricos a la resolución de problemas, y conecte los contenidos del aula, con las situaciones de la vida real (Fensham, 2009), en una amplia variedad de contextos y escenarios. En definitiva, gestionando estrategias de reflexión (Lupión y Blanco, 2016) que permitan al alumnado, conectar con su identificación de la ciencia y los valores de ésta, en aras al fomento de futuras vocaciones científico-técnicas.

Desde este planteamiento educativo, disponer de un adecuado diagnóstico de la situación de partida en las percepciones del alumnado respecto a los predictores de estas vocaciones es un planteamiento de interés, en el objetivo estratégico del diseño de futuras situaciones de enseñanza, para una intervención docente dirigida a su promoción.

En nuestro interés por conocer las percepciones del alumnado de secundaria de ciencias hacia la CyT y su vocación hacia ellas, iniciamos un primer estudio (Lupión-Cobos, Franco-Mariscal y Girón-Gambero, 2019), sobre ciertos aspectos considerados en la literatura (Vázquez y Manassero 2008, 2009; Sjøberg y Schreiner 2005, Pérez 2013), como predictores de vocaciones científicas: la aceptación de la indagación como estilo de pensamiento, el interés por estudiar carreras científicas y, el disfrute en las clases de ciencias. En la investigación que aquí presentamos, continuamos dicho estudio, centrado ahora, en analizar otros aspectos relevantes sobre estos predictores, relativos a las percepciones de las que parte el alumnado de entre 15 a 17 años, al iniciar sus estudios correspondientes en esta etapa, respecto a aspectos sobre la función social de la ciencia.

\section{OBJETIVOS DE LA INVESTIGACIÓN}

Este estudio forma parte de una investigación más amplia que estudia las actitudes que presentan los y las estudiantes españoles de secundaria en torno a la indagación científica. En concreto, tiene por objetivo conocer la percepción social que el alumnado tiene de la ciencia, centrándose en las siguientes preguntas de investigación:

- Cuestión 1: ¿Cuáles son las percepciones del alumnado de secundaria hacia estos tres aspectos sociales de la ciencia: ¿sus implicaciones sociales, la adopción de actitudes científicas y el interés por la ciencia en el tiempo de ocio? 
- Cuestión 2: ¿Cómo valora el alumnado estos aspectos en función de su género y nivel educativo (educación obligatoria y postobligatoria)?

\section{MÉTODO}

\subsection{Participantes}

La muestra de la investigación estuvo formada por 158 estudiantes de educación secundaria de edades comprendidas entre 15 y 17 años que cursaban sus estudios en siete institutos de secundaria de centros públicos españoles de nivel socioeconómico medio. La muestra constaba de 64 estudiantes pertenecientes a la educación obligatoria ( $4^{\circ}$ ESO; $15-16$ años) y 95 estudiantes de la educación postobligatoria ( $1{ }^{\circ}$ de Bachillerato modalidad científica,16-17 años). El 62,3\% eran chicas y el 37,7\% chicos. La mayoría del alumnado había seguido una enseñanza tradicional y no había recibido una formación específica sobre investigaciones escolares.

\subsection{Instrumento de recogida de datos}

El instrumento utilizado en este trabajo fue la prueba TOSRA (Test of Science Related Attitudes), con validación internacional (Fraser, 1981; Cheung, 2009; Welch, 2010) y que recoge información sobre la percepción del alumnado de ciencias sobre diferentes aspectos de la investigación científica. Esta prueba incluye siete dimensiones: implicaciones sociales de la ciencia (S), visión de la vida de los científicos $(\mathrm{N})$, actitudes hacia la indagación científica (I), adopción de actitudes científicas (A), disfrute de las clases de ciencia (E), interés por la ciencia en el tiempo de ocio (L), e interés por estudiar carreras científicas (C).

Cada dimensión se compone de 10 enunciados, por lo que la prueba TOSRA administrada presentaba 70 ítems (Fraser, 1981). Cada enunciado, se ofreció en inglés para no diferir del original, siendo traducido con el alumnado para garantizar la correcta comprensión de su contenido (El listado completo en su enunciado en inglés y español, puede verse publicado en Navarro et al., 2016), estando cada enunciado planteado en 5 ítems, en sentido positivo (a favor de la dimensión) y otros 5 ítems, en sentido negativo (en contra de la dimensión) en una escala Likert de elección de 5 puntos (1: strongly disagree (SD), 2: disagree (D), 3: not sure (N), 4: agree (A), 5: strongly agree (SA)). De este modo, la valoración que puede obtener un estudiante en cada dimensión se sitúa entre 10 y 50 puntos. El instrumento incluía también preguntas sobre aspectos sociodemográficos (edad, sexo, curso, centro de estudios).

Los resultados obtenidos de la prueba relacionados con la percepción social de la ciencia (Autores, 2019), objeto de este trabajo, se centra en las tres dimensiones estrechamente vinculadas con ella y que se asocian, a las dimensiones S, A y L del instrumento. Las subescalas del mismo, presentan una fiabilidad que oscila entre 0,64 y 0,93, y la fiabilidad global es adecuada y de 0,90 (Fraser, 1981; Unfried, Faber, Stanhope y Wiebe, 2015).

\subsection{Análisis estadístico}

Se realizó un análisis descriptivo de los datos con el programa estadístico SPSS 21.0. Se calcularon medidas de posición central y de dispersión (frecuencia, media, error estándar de la media y desviación estándar típica). Las características psicométricas de nuestro estudio son adecuadas, siendo el coeficiente alfa de Cronbach de las dimensiones S, A y L objeto de estudio 0,742, 0,619 y 0,865 , respectivamente. De la misma manera, el análisis de componentes principales muestra que la prueba tiene una varianza total explicada de 68,453. Para conocer las diferencias de percepciones del alumnado en función del género y nivel educativo se aplicó la prueba t-student para muestras independientes, comprobándose en todos los casos la homocedasticidad de las varianzas. 


\section{RESULTADOS Y DISCUSIÓN}

Se presenta un análisis de las percepciones encontradas en el alumnado, en primer lugar, según los tres aspectos estudiados y seguidamente, en función de su género y nivel educativo.

\subsection{Diferencias de las percepciones en las dimensiones estudiadas}

El presente apartado recoge los resultados para cada una de las dimensiones analizadas. Para ello, se presentan los descriptivos básicos para cada ítem y el total de la dimensión. Los resultados se analizan sumando, por un lado, las opciones 1 y 2 (SD y D) de la escala Likert, y, por otra parte, las opciones 4 y 5 (A y SA), lo que permitirá establecer una comparación entre los y las estudiantes a favor y en contra de cada ítem. Se considera que manifiestan una actitud positiva hacia el ítem propuesto si las respuestas mayoritarias se sitúan en las opciones 4 y 5 para ítems formulados en sentido positivo, y en las opciones 1 y 2 para ítems en sentido negativo. El cálculo de la media total para cada dimensión se realiza sumando los parciales de los valores medios de los ítems positivos y los valores medios convertidos para este propósito por el programa de los ítems negativos. Las tablas recogen en negrita el bloque de respuestas mayoritarias.

\section{Percepción del alumnado hacia la implicación social de la ciencia (dimensión S)}

Los resultados de la dimensión S se recogen en la figura 1, que muestra el histograma para el total de la dimensión, y en la tabla 1 que presenta los porcentajes de elección de cada opción junto con los estadísticos descriptivos básicos para los ítems y el total de la dimensión.

TABLA 1. Ítems y estadísticos descriptivos de la dimensión S sobre implicaciones sociales de la ciencia

\begin{tabular}{|c|c|c|c|c|c|c|c|c|c|c|}
\hline \multirow[b]{2}{*}{ Ítem } & \multicolumn{5}{|c|}{ Porcentajes } & \multirow[b]{2}{*}{$\bar{x}$} & \multirow{2}{*}{$\begin{array}{c}\bar{x} \\
\text { conv }\end{array}$} & \multirow[b]{2}{*}{ EEM } & \multirow[b]{2}{*}{$\mathrm{Sx}$} & \multirow[b]{2}{*}{ Sentido } \\
\hline & $\begin{array}{c}1 \\
(\mathrm{SD})\end{array}$ & $\begin{array}{c}2 \\
\text { (D) }\end{array}$ & $\begin{array}{c}3 \\
(\mathrm{~N})\end{array}$ & $\begin{array}{c}4 \\
\text { (A) }\end{array}$ & $\begin{array}{c}5 \\
(\mathrm{SA}) \\
\end{array}$ & & & & & \\
\hline $\begin{array}{l}\text { 01. El dinero que } \\
\text { se invierte en } \\
\text { ciencia es dinero } \\
\text { bien invertido }\end{array}$ & 0 & 1,9 & 7,0 & 34,8 & 56,3 & 4,46 & & 0,057 & 0,710 & + \\
\hline $\begin{array}{l}\text { 08. La ciencia es } \\
\text { el peor enemigo } \\
\text { del hombre }\end{array}$ & 69,0 & 14,6 & 8,9 & 5,7 & 1,9 & 1,57 & 4,43 & 0,079 & 0,999 & - \\
\hline $\begin{array}{l}\text { 15. El dinero } \\
\text { público dedicado } \\
\text { a la ciencia en los } \\
\text { últimos años ha } \\
\text { sido utilizado } \\
\text { con inteligencia }\end{array}$ & 5,7 & 10,8 & 32,9 & 29,7 & 20,9 & 3,49 & & 0,088 & 1,110 & + \\
\hline $\begin{array}{lr}22 . & \text { Los } \\
\text { descubrimientos } \\
\text { científicos } & \text { están } \\
\text { causando más } \\
\text { daño } & \text { que } \\
\text { beneficio } & \\
\end{array}$ & 43,0 & 25,3 & 19,0 & 8,2 & 4,4 & 2,06 & 3,94 & 0,093 & 1,163 & - \\
\hline $\begin{array}{l}\text { 29. El gobierno } \\
\text { debe gastar más } \\
\text { dinero en } \\
\text { investigación } \\
\text { científica }\end{array}$ & 0 & 0 & 13,9 & 40,5 & 45,6 & 4,32 & & 0,056 & 0,706 & + \\
\hline
\end{tabular}




\begin{tabular}{|c|c|c|c|c|c|c|c|c|c|c|}
\hline $\begin{array}{l}36 . \text { Se están } \\
\text { construyendo } \\
\text { demasiados } \\
\text { laboratorios a } \\
\text { costa de la } \\
\text { disminución de } \\
\text { la inversión para } \\
\text { el resto de la } \\
\text { educación }\end{array}$ & 10,1 & 19,0 & 46,2 & 18,4 & 6,3 & 2,92 & 3,08 & 0,081 & 1,016 & - \\
\hline $\begin{array}{l}\text { 43. La ciencia } \\
\text { contribuye a } \\
\text { mejorar la } \\
\text { calidad de vida }\end{array}$ & 0 & 1,3 & 10,1 & 39,2 & 49,4 & 4,37 & & 0,057 & 0,717 & + \\
\hline $\begin{array}{l}\text { 50. Nuestro país } \\
\text { está gastando } \\
\text { demasiado } \\
\text { dinero en ciencia }\end{array}$ & 31,6 & 25,9 & 29,7 & 9,5 & 3,2 & 2,27 & 3,73 & 0,088 & 1,103 & - \\
\hline $\begin{array}{l}57 . \text { La ciencia } \\
\text { puede ayudar a } \\
\text { hacer del mundo } \\
\text { un lugar mejor }\end{array}$ & 0 & 0,6 & 5,7 & 29,7 & 63,9 & 4,57 & & 0,050 & 0,632 & + \\
\hline $\begin{array}{lr}64 . \quad \text { El } & \text { dinero } \\
\text { usado } & \text { en } \\
\text { proyectos } & \\
\text { científicos } & \text { es } \\
\text { dinero } & \\
\text { desperdiciado } & \\
\end{array}$ & 53,2 & 25,3 & 13,3 & 4,4 & 3,8 & 1,80 & 4,2 & 0,085 & 1,073 & - \\
\hline $\begin{array}{l}\text { Total } \\
\text { Dimensión S }\end{array}$ & & & & & & 40,59 & 0,412 & 5,174 & & \\
\hline
\end{tabular}

$\bar{X}$ : Media; EEM: Error estándar de la media; SX: desviación estándar típica Fuente: elaboración propia

GRÁFICO 1. Diagrama de barras de los ítems positivos y negativos que conforman la dimensión S sobre implicaciones sociales de la ciencia

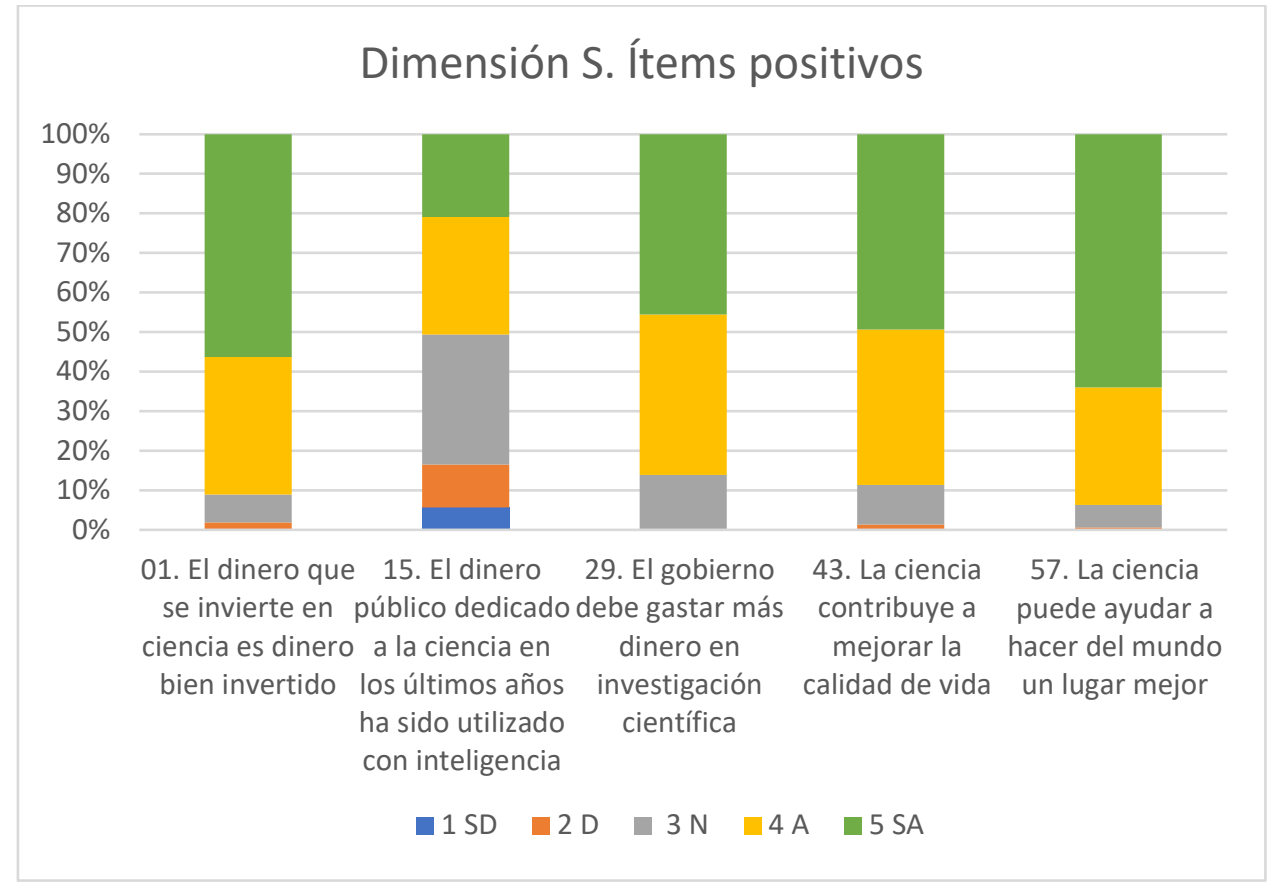




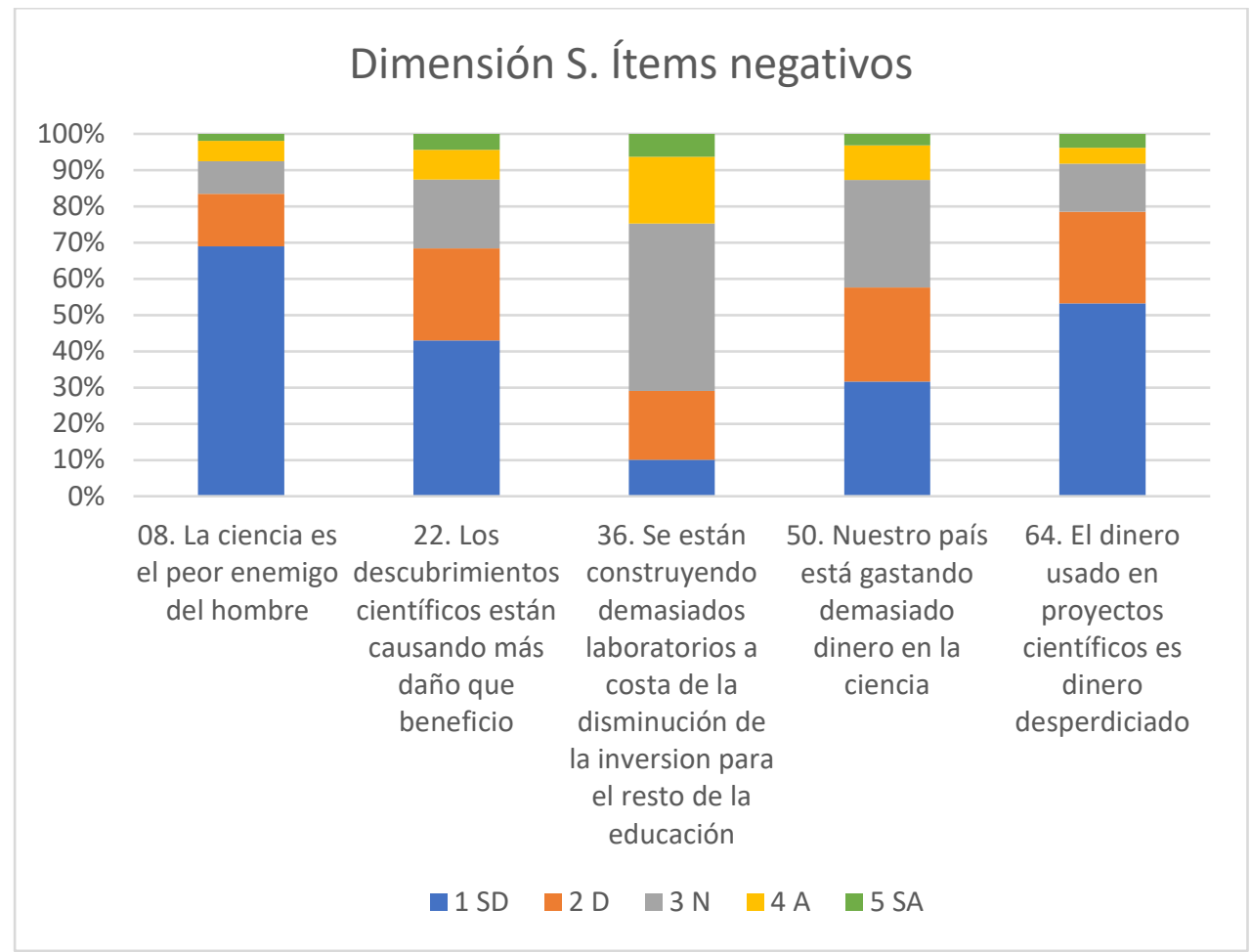

Fuente: elaboración propia

La media global de esta dimensión (sobre 50) (figura 1) $(\overline{\mathrm{X}}=40,59 ; \mathrm{Sx}=5,26)$, con mínimo en 22 puntos y máximo en 50 puntos, parece mostrar que el alumnado manifiesta una tendencia actitudinal positiva en sus percepciones sobre las implicaciones sociales de la ciencia.

Los datos de los ítems parecen indicar una concienciación ciudadana en los diferentes aspectos cuestionados (a excepción del ítem 36) al haber valorado los estudiantes de forma mayoritaria el bloque de respuestas a favor del ítem (opciones 4 y 5 para ítems positivos y opciones 1 y 2 para ítems negativos). Cabe destacar que seis de estos ítems supera el 78\%, siendo el ítem 57 "La ciencia puede ayudar a hacer del mundo un lugar mejor" el mejor valorado $(\overline{\mathrm{x}}=4,57 ; \mathrm{Sx}=0,632)$.

Mención especial merece el ítem 36, "Se están construyendo demasiados laboratorios a costa de la disminución de la inversión para el resto de la educación” $(\overline{\mathrm{X}}=2,92 ; \mathrm{Sx}=1,016)$, en el que prácticamente la mitad de los sujetos están indecisos. Este dato pudiera justificarse debido a que, aunque el alumnado está de acuerdo con que se realice inversión en ciencia, tal y como recoge el ítem 1, "El dinero que se invierte en ciencia es dinero bien invertido" $(\overline{\mathrm{x}}=4,46 ; \mathrm{Sx}=0,710)$, la comunicación sobre temas científicos y la inversión en investigación no se difunde a la sociedad, o bien realmente es muy pobre y por ello no se evidencia. De ahí que el alumnado no tenga suficiente criterio para su valoración. Este dato se ve refrendado por el ítem 15, "El dinero público dedicado a la ciencia en los últimos años ha sido utilizado con inteligencia" que presenta el porcentaje menos valorado $(50,6 \%)$.

Percepción del alumnado hacia la adopción de actitudes cientificas (dimensión A)

La tabla 2 y la figura 2 recogen los datos obtenidos y el histograma respectivamente para la dimensión A, sobre la adopción de actitudes científicas por parte del alumnado. 
TABLA 2. Estadísticos descriptivos básicos para la dimensión A sobre adopción de actitudes científicas

\begin{tabular}{|c|c|c|c|c|c|c|c|c|c|c|}
\hline \multirow[b]{2}{*}{ Ítem } & \multicolumn{5}{|c|}{ Porcentajes } & \multirow[b]{2}{*}{$\bar{x}$} & \multirow[b]{2}{*}{$\begin{array}{c}\bar{x} \\
\text { conv. }\end{array}$} & \multirow[b]{2}{*}{ EEM } & \multirow[b]{2}{*}{ Sx } & \multirow[b]{2}{*}{ Sentido } \\
\hline & $\begin{array}{c}1 \\
(\mathrm{SD}) \\
\end{array}$ & $\begin{array}{c}2 \\
(\mathrm{D})\end{array}$ & $\begin{array}{c}3 \\
(\mathrm{~N}) \\
\end{array}$ & $\begin{array}{c}4 \\
(\mathrm{~A}) \\
\end{array}$ & $\begin{array}{c}5 \\
(\mathrm{SA}) \\
\end{array}$ & & & & & \\
\hline $\begin{array}{l}\text { 04. Me gusta leer sobre } \\
\text { temas que no están de } \\
\text { acuerdo con mis ideas }\end{array}$ & 1,9 & 15,8 & 24,7 & 40,5 & 17,1 & 3,55 & & 0,081 & 1,013 & + \\
\hline $\begin{array}{l}11 . \text { No me gusta } \\
\text { repetir } \\
\text { experimentos para } \\
\text { comprobar que dan los } \\
\text { mismos resultados }\end{array}$ & 15,8 & 34,2 & 24,1 & 15,8 & 10,1 & 2,70 & 3,3 & 0,096 & 1,208 & - \\
\hline $\begin{array}{l}\text { 18. Tengo curiosidad } \\
\text { acerca del mundo en } \\
\text { que vivimos }\end{array}$ & 0,6 & 1,9 & 3,2 & 33,5 & 60,8 & 4,52 & & 0,057 & 0,711 & + \\
\hline $\begin{array}{l}25 . \text { No es importante } \\
\text { investigar sobre cosas } \\
\text { nuevas. }\end{array}$ & 59,5 & 24,7 & 3,8 & 8,9 & 3,2 & 1,72 & 4,28 & 0,087 & 1,095 & - \\
\hline $\begin{array}{l}\text { 32. Me gusta escuchar } \\
\text { a las personas cuyas } \\
\text { opiniones son } \\
\text { diferentes a las mías }\end{array}$ & 1,3 & 3,8 & 7,6 & 57,6 & 29,7 & 4,11 & & 0,063 & 0,795 & + \\
\hline $\begin{array}{l}\text { 39. Me parece aburrido } \\
\text { oír ideas nuevas }\end{array}$ & 38,0 & 44,9 & 10,8 & 3,8 & 2,5 & 1,88 & 4,12 & 0,074 & 0,926 & - \\
\hline $\begin{array}{l}46 . \quad \text { En los } \\
\text { experimentos } \\
\text { científicos me gusta } \\
\text { usar métodos que no he } \\
\text { usado antes }\end{array}$ & 0 & 4,4 & 19,0 & 50,0 & 26,6 & 3,99 & & 0,063 & 0,798 & + \\
\hline $\begin{array}{l}\text { 53. No estoy dispuesto } \\
\text { a cambiar mis ideas } \\
\text { aunque la realidad } \\
\text { muestre que éstas no } \\
\text { tienen suficiente base }\end{array}$ & 22,8 & 20,3 & 24,7 & 24,1 & 8,2 & 2,75 & 3,25 & 0,102 & 1,277 & - \\
\hline $\begin{array}{l}\text { 60. En los } \\
\text { científicos rormes } \\
\text { tanto los reporto } \\
\text { esperados como los } \\
\text { inesperados } \\
\text { indodos }\end{array}$ & 2,5 & 10,1 & 39,2 & 34,8 & 13,3 & 3,46 & & 0,074 & 0,935 & + \\
\hline $\begin{array}{l}\text { 67. No me gusta } \\
\text { escuchar las opiniones } \\
\text { de otras personas }\end{array}$ & 45,6 & 38,0 & 8,9 & 3,8 & 3,8 & 1,82 & 4,18 & 0,080 & 1,006 & - \\
\hline Total Dimensión A & & & & & & & ,76 & 0,375 & 4,713 & \\
\hline
\end{tabular}

$\overline{\mathrm{X}}$ : Media; EEM: Error estándar de la media; SX: desviación estándar típica

Fuente: elaboración propia 
GRÁFICO 2. Diagrama de barras de los ítems positivos y negativos que conforman la dimensión A sobre implicaciones sociales de la ciencia
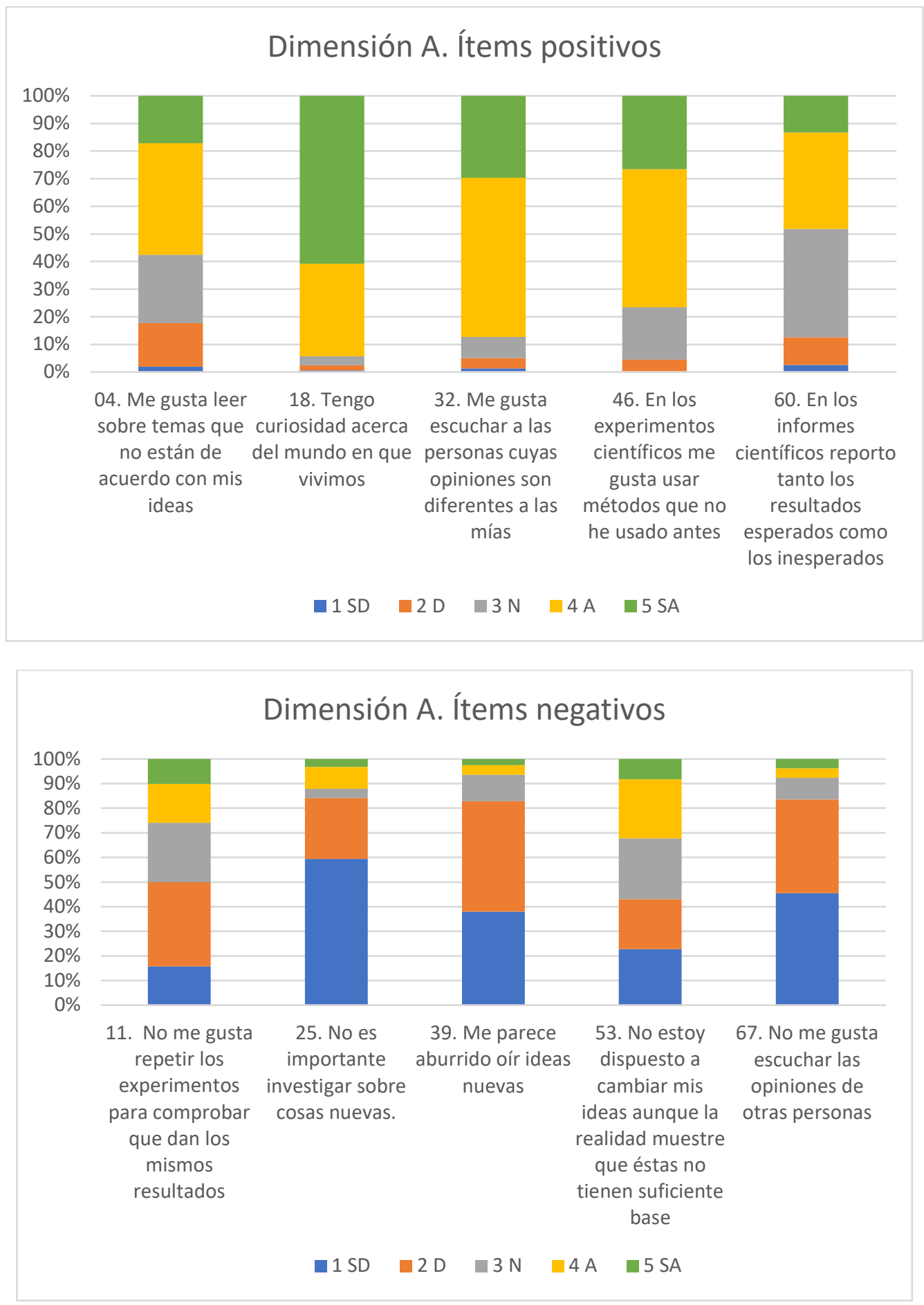

Fuente: elaboración propia

La media global de esta dimensión $(\overline{\mathrm{x}}=38,76 ; \mathrm{Sx}=4,71)$ parece mostrar una tendencia actitudinal positiva en sus adopciones de actitudes científicas, situándose el mínimo y el máximo en 26 puntos y 50 puntos, respectivamente.

Los datos obtenidos (tabla 2) parecen indicar buenos resultados, al situarse en todos los ítems las percepciones del alumnado en el bloque de respuestas a favor de la afirmación. Seis de los ítems superan el 76\%, siendo el ítem 18, "Tengo curiosidad acerca del mundo en que vivimos" el más 
valorado, por el 94,3\% de los estudiantes (valores 4 y 5) $(\overline{\mathrm{X}}=4,52 ; \mathrm{Sx}=0,71)$, no solo en esta dimensión sino también en todas las analizadas. Cabe destacar la lectura del ítem 32, "Me gusta escuchar a personas cuyas opiniones son distintas a las mías" $(\overline{\mathrm{X}}=4,11 ; \mathrm{Sx}=0,795)$, que indica una conciencia social adecuada, en lo que a escuchar a los demás se refiere, pero sin embargo, al combinarlo con el ítem 53, uno de los que recibe una menor valoración "No estoy dispuesto a cambiar mis ideas aunque la realidad muestre que éstas no tienen suficiente base" $(43,1 \%)(\overline{\mathrm{x}}=2,75 ; \mathrm{Sx}=$ $1,27)$, muestra un indicio de que no han logrado interiorizar claramente la reflexión y autocrítica en la toma de decisiones, propias del pensamiento científico, más allá de la interacción social respetuosa.

Destaca el ítem 60, "En los informes científicos reporto tanto los resultados esperados como los inesperados" ( $\overline{\mathrm{X}}=3,46$; Sx $=0,93)$, que, a pesar de ser favorable, sobresale un porcentaje importante de estudiantes indecisos (39\%). Entendemos que esto se debe a no tener asumida la ética científica en evidenciar los resultados que la experimentación muestra, sean los esperados o no, lo que induce a pensar que este aspecto no está muy incorporado en los programas educativos y, por tanto, no llega a trascender a la ciudadanía.

\section{Percepción del alumnado hacia su interés por la ciencia en el tiempo de ocio (dimensión L)}

Para la dimensión L, la figura 3 y la tabla 3 recogen los resultados obtenidos. Del histograma (figura 3 ) se deduce que la media global de esta dimensión $(\bar{X}=35,91 ; \mathrm{Sx}=7,37)$ muestra de nuevo una tendencia actitudinal positiva, en este caso, hacia el interés del alumnado por la ciencia en su tiempo libre. No obstante, la campana de Gauss está en esta ocasión más desplazada hacia la izquierda al situarse el mínimo y máximo en 16 y 50 puntos, respectivamente.

TABLA 3. Estadísticos descriptivos básicos para la dimensión L sobre interés por la ciencia en el tiempo de ocio

\begin{tabular}{|c|c|c|c|c|c|c|c|c|c|c|}
\hline \multirow[b]{2}{*}{ Ítem } & \multicolumn{5}{|c|}{ Porcentajes } & \multirow[b]{2}{*}{$\bar{x}$} & \multirow[b]{2}{*}{$\bar{x}$ conv. } & \multirow[b]{2}{*}{ EEM } & \multirow[b]{2}{*}{$\mathrm{Sx}$} & \multirow[b]{2}{*}{ Sentido } \\
\hline & $\begin{array}{c}1 \\
(\mathrm{SD})\end{array}$ & $\begin{array}{c}2 \\
(\mathrm{D})\end{array}$ & $\begin{array}{c}3 \\
(\mathrm{~N}) \\
\end{array}$ & $\begin{array}{c}4 \\
(\mathrm{~A}) \\
\end{array}$ & $\begin{array}{c}5 \\
(\mathrm{SA}) \\
\end{array}$ & & & & & \\
\hline $\begin{array}{l}\text { 06. Me gustaría pertenecer a } \\
\text { un club de la ciencia }\end{array}$ & 4,4 & 8,9 & 28,5 & 39,9 & 18,4 & 3,59 & & 0,082 & 1,029 & + \\
\hline $\begin{array}{l}\text { 13. Me aburro cuando veo } \\
\text { en mi casa programas } \\
\text { científicos en la televisión }\end{array}$ & 32,9 & 36,7 & 13,3 & 13,9 & 3,2 & 2,18 & 3,82 & 0,090 & 1,132 & - \\
\hline $\begin{array}{l}20 . \text { Me gustaría recibir } \\
\text { como regalo un libro de } \\
\text { ciencias o un instrumento } \\
\text { científico }\end{array}$ & 6,3 & 12,0 & 31,0 & 36,7 & 13,9 & 3,40 & & 0,085 & 1,070 & + \\
\hline $\begin{array}{l}\text { 27. No me gusta leer libros } \\
\text { de ciencias durante mis } \\
\text { vacaciones }\end{array}$ & 13,9 & 30,4 & 22,8 & 20,9 & 12,0 & 2,87 & 3,13 & 0,099 & 1,242 & - \\
\hline $\begin{array}{l}\text { 34. Me gustaría hacer } \\
\text { experimentos científicos en } \\
\text { mi casa }\end{array}$ & 2,5 & 6,3 & 22,2 & 44,3 & 24,7 & 3,82 & & 0,076 & 0,961 & + \\
\hline $\begin{array}{l}\text { 41. Sería aburrido conversar } \\
\text { con los amigos sobre ciencia } \\
\text { después del colegio }\end{array}$ & 24,1 & 35,4 & 25,3 & 13,3 & 1,9 & 2,34 & 3,66 & 0,083 & 1,044 & - \\
\hline $\begin{array}{l}\text { 48. Disfrutaría con tener un } \\
\text { trabajo en un laboratorio de } \\
\text { ciencias durante mis } \\
\text { vacaciones escolares }\end{array}$ & 7,6 & 10,8 & 29,7 & 29,1 & 22,8 & 3,49 & & 0,094 & 1,177 & + \\
\hline
\end{tabular}




\begin{tabular}{|l|l|l|l|l|l|l|l|l|l|l|}
$\begin{array}{l}\text { 55. Sería aburrido escuchar } \\
\text { un programa de ciencia en la } \\
\text { radio }\end{array}$ & $\mathbf{1 9 , 0}$ & $\mathbf{3 8 , 6}$ & 25,3 & 11,4 & 5,7 & 2,46 & 3,54 & 0,087 & 1,098 & - \\
\hline $\begin{array}{l}\text { 62. Me gustaría visitar un } \\
\text { museo de ciencias durante } \\
\text { mis fines de semana }\end{array}$ & 4,4 & 7,6 & 18,4 & $\mathbf{4 1 , 8}$ & $\mathbf{2 7 , 8}$ & 3,81 & & 0,085 & 1,066 & + \\
\hline $\begin{array}{l}\text { 69. No me gusta leer } \\
\text { artículos periodísticos sobre } \\
\text { ciencia }\end{array}$ & $\mathbf{2 3 , 4}$ & $\mathbf{3 9 , 9}$ & 19,0 & 12,7 & 5,1 & 2,36 & 3,64 & 0,089 & 1,124 & - \\
\hline
\end{tabular}

$\overline{\mathrm{X}}$ : Media; EEM: Error estándar de la media; SX: desviación estándar típica

Fuente: elaboración propia

GRÁFICO 3. Diagrama de barras de los ítems positivos y negativos que conforman la dimensión L sobre implicaciones sociales de la ciencia
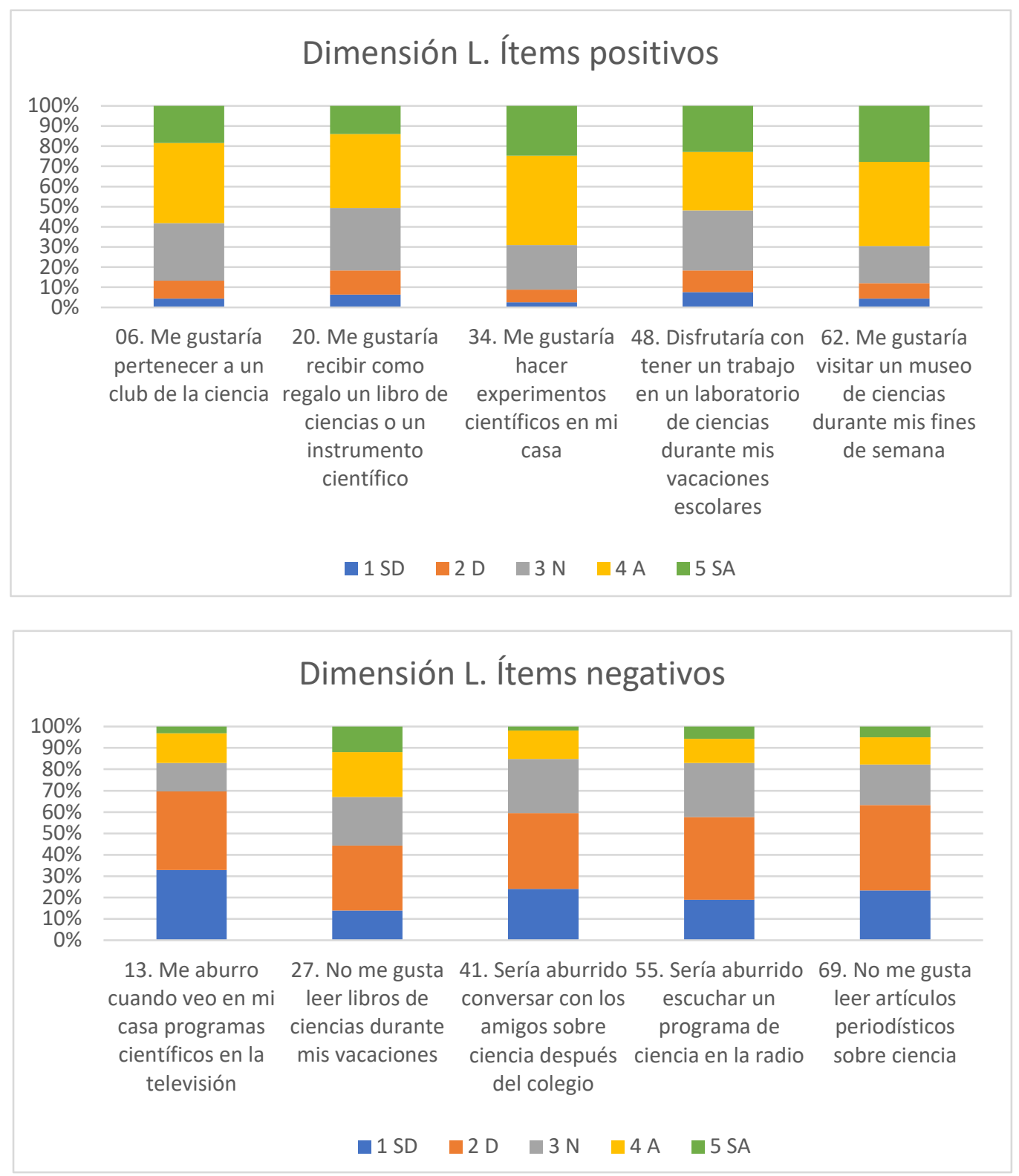

Fuente: elaboración propia 
A pesar de que los resultados son favorables (gráfico 3) al posicionarse la mayoría del alumnado con una actitud positiva en todos los ítems, en esta ocasión deben considerarse más modestos que los alcanzados en las anteriores dimensiones debido a que los porcentajes no son tan elevados y la suma de los valores 4 y 5 para ninguno de los ítems logra superar el 70\%. Destaca también el elevado porcentaje de indecisos (31\%) en el ítem 20, "Me gustaría recibir como regalo un libro de ciencias o un instrumento científico" $(\overline{\mathrm{x}}=3,40 ; \mathrm{Sx}=1,07)$, lo que podría reflejar la ausencia de una futura vocación científica, pues los adolescentes a menudo muestran su vocación con juegos o aficiones lo que nos sugiere la importancia de incorporar metodologías activas como la gamificación entre las estrategias usadas en el aula para despertar el interés hacia el aprendizaje (Fernández Sierra et al., 2019).

\section{Análisis conjunto de las tres dimensiones}

Una vez analizadas las tres dimensiones, los datos parecen revelar la mayor tendencia actitudinal del alumnado en el siguiente orden: implicaciones sociales de la ciencia $(S)(\bar{X}=40,49$; $\mathrm{Sx}=5,26)$, adopción de actitudes científicas $(\mathrm{A})(\overline{\mathrm{X}}=38,76 ; \mathrm{Sx}=4,71)$ e interés por la ciencia en el tiempo de ocio (L) $(\overline{\mathrm{X}}=35,91 ; \mathrm{Sx}=7,37)$. Este orden se puede relacionar con una mayor interiorización de una actitud personal que responda a que el/la estudiante haya asumido en su vida diaria un comportamiento más coherente frente a una verdadera valoración o reconocimiento de la actividad científica. Así, mientras que las dimensiones A y L representan comportamientos adoptados por la persona a ejercer actuaciones concretas en su vida, es decir, se trataría de una intervención en la acción, los ítems asociados en la dimensión $\mathrm{S}$, están referidos a valoraciones sobre consideraciones, que no implican que el sujeto se haya implicado en una intervención en su propia actuación.

Al analizar de manera individual todos los ítems valorados, se encuentra que los ítems que alcanzan mayor porcentaje de valoración positiva (ítem 18, "Tengo curiosidad acerca del mundo en que vivimos") y menor (ítem 53, "No estoy dispuesto a cambiar mis ideas, aunque la realidad muestre que éstas no tienen suficiente base”) pertenecen precisamente a la misma dimensión sobre adopción de actitudes científicas, que ocupa el segundo lugar.

\subsection{Diferencias de las percepciones por género y nivel educativo}

\section{Diferencias por género}

En la dimensión $\mathrm{S}$ los chicos presentaron una media de 39,66 $(\mathrm{Sx}=6,00)$ mientras que para las chicas fue 41,14 ( $\mathrm{Sx}=4,55)$ (Tabla 4). Para la dimensión A la media de los chicos fue de 37,53 ( $\mathrm{Sx}$ $=4,75)$ y para las chicas, 39,49 $(\mathrm{Sx}=4,55)$. En la dimensión L la media de los chicos y chicas fueron $33,83(\mathrm{Sx}=7,97)$ y $37,14(\mathrm{Sx}=6,74)$, respectivamente. La prueba $\mathrm{t}$ de student para muestras independientes mostró diferencias estadísticamente significativas entre chicos y chicas, a favor de estas últimas en las dimensiones $\mathrm{A}\left(\mathrm{t}_{(2,156)}=-2,59 ; \mathrm{p}<, 05\right)$ y $\mathrm{L}\left(\mathrm{t}_{(2,156)}=-2,79 ; \mathrm{p}<, 05\right)$, pero no en la dimensión $\mathrm{S}\left(\mathrm{t}_{(2,97.80)}=-1,63 ; \mathrm{p}>, 05\right)$ (tabla 4), lo que pone de manifiesto que las chicas parecen adoptar mayores actitudes científicas y tienen más interés por la ciencia en el tiempo de ocio que los chicos.

\section{Diferencias por nivel educativo}

El alumnado de $1^{\circ}$ de Bachillerato presentaron en todas las dimensiones medias mayores que los de $4^{\circ}$ ESO tabla 4), encontrándose las mayores diferencias en la dimensión L ( $\overline{\mathrm{x}}=33,59 ; \mathrm{Sx}=$ 7,58 para $4^{\circ}$ ESO y $\bar{x}=37,48 ; \mathrm{Sx}=6,84$, para $1^{\circ}$ Bachilllerato). Esto puede deberse a que el alumnado de Bachillerato, ha alcanzado un grado de madurez mayor, sobrepasando los 16 años y estando en el 
primer curso de una enseñanza no obligatoria lo que indica una mayor consistencia a la hora de establecer sus gustos o preferencias en el tiempo de ocio.

TABLA 4. Prueba $t$ de student para muestras independientes según género

\begin{tabular}{cccccccccc} 
Dimensión & Género & $\mathrm{N}$ & $\overline{\mathrm{X}}$ & $\mathrm{Sx}$ & EEM & $\mathrm{F}$ & $\mathrm{P}$ & $\mathrm{t}$ & g.l. \\
\hline S & Chico & 59 & 39,66 & 6,002 & 0,781 & 6,341 & 0,013 & $-1,635$ & $\begin{array}{c}97,80 \\
\end{array}$ \\
& Chica & 99 & 41,14 & 4,554 & 0,458 & & & & \\
A & Chico & 59 & 37,53 & 4,754 & 0,619 & 0,216 & 0,643 & $-2,587$ & 156 \\
& Chica & 99 & 39,49 & 4,554 & 0,458 & & & & \\
L & Chico & 59 & 33,83 & 7,974 & 1,038 & 1,362 & 0,245 & $-2,788$ & 156 \\
& Chica & 99 & 37,14 & 6,736 & 0,677 & & & & \\
\hline
\end{tabular}

$\mathrm{N}$ : Muestra; $\overline{\mathrm{x}}$ : media; Sx: Desviación típica; EEM: Error estándar de la media; F: Estadístico de Levene de calidad de varianzas; t: Estadístico t de student para muestras independientes; g.1.: Grados de libertad; p: Nivel de significación:

TABLA 5. Prueba $t$ de student para muestras independientes según curso

\begin{tabular}{cccccccccc} 
Dimensión & Grado & $\mathrm{N}$ & $\overline{\mathrm{x}}$ & $\mathrm{Sx}$ & $\mathrm{EEM}$ & $\mathrm{F}$ & $\mathrm{p}$ & $\mathrm{t}$ & $\mathrm{g} .1$. \\
\hline $\mathrm{S}$ & $4^{\circ} \mathrm{ESO}$ & 64 & 39,19 & 5,294 & 0,662 & 0,052 & 0,820 & $-2,873$ & 156 \\
& $1^{\circ} \mathrm{Bach}$ & 94 & 41,54 & 4,892 & 0,505 & & & & \\
$\mathrm{~A}$ & $4^{\circ} \mathrm{ESO}$ & 64 & 38,03 & 4,567 & 0,571 & 0,150 & 0,699 & $-1,611$ & 156 \\
& $1^{\circ} \mathrm{Bach}$ & 94 & 39,26 & 4,770 & 0,492 & & & & \\
L & $4^{\circ} \mathrm{ESO}$ & 64 & 33,59 & 7,580 & 0,947 & 1,097 & 0,296 & $-3,355$ & 156 \\
& $1^{\circ} \mathrm{Bach}$ & 94 & 37,48 & 6,836 & 0,705 & & & & \\
\hline
\end{tabular}

N: Muestra; $\overline{\mathrm{X}}$ : media; Sx: Desviación típica; EEM: Error estándar de la media; F: Estadístico de Levene de calidad de varianzas; t: Estadístico t de student para muestras independientes; g.1.: Grados de libertad; p: Nivel de significación.

Fuente: elaboración propia

La t de student mostró sólo diferencias estadísticamente significativas en alumnado de $4^{\circ} \mathrm{ESO}$ y $1^{\mathrm{o}}$ Bachillerato en las dimensiones $\mathrm{S}\left(\mathrm{t}_{(2,156)}=-2,87 ; \mathrm{p}<, 05\right)$ y $\mathrm{L}\left(\mathrm{t}_{(2,156)}=-3,35 ; \mathrm{p}<, 05\right)$, a favor de estos últimos (tabla 5). Resultados que evidencian la tendencia del estudiantado de Bachillerato a implicarse socialmente en aspectos relativos a la ciencia y a interesarse por ella en su tiempo de ocio. Estos datos parecen sugerir una incidencia efectiva de la incorporación de contenidos actitudinales en los valores que dicho alumnado asocia al proceso de formación en educación científica, al pasar de una educación obligatoria a otra postobligatoria elegida de forma opcional en su orientación científica.

Estos resultados contrastan con la aparente tendencia de que a medida que se avanza en el nivel educativo, disminuye la valoración actitudinal hacia la ciencia del estudiantado, lo que nos hace pensar en la incidencia de otros factores como la relación inversa entre el grado de desarrollo del país y las actitudes positivas hacia la ciencia en jóvenes de secundaria (Sjøberg y Schreiner, 2005, 2007), y en concreto en las diferencias existentes entre los niveles de desarrollo del entorno geográfico en 
relación con las actitudes, recogida en la literatura (Gil, 2012), confirmando la existencia de otros factores en las percepciones del alumnado o en su interés por las ciencias.

\section{CONCLUSIONES Y PERSPECTIVAS DE FUTURO}

Los resultados alcanzados en este estudio deben contemplarse con las limitaciones asociadas al tamaño de la muestra y su composición, dado que su selección no ha sido aleatoria y, por tanto, la generalización al comportamiento de estudiantes de estas etapas educativas, debe ser tratada con la cautela debida. Asimismo, la naturaleza del diseño del estudio hace contemplar el análisis de la evolución de las percepciones del alumnado, con carácter limitado.

De esta investigación se pueden extraer diversas conclusiones sobre las percepciones del alumnado del estudio, respecto a sus percepciones en las tres dimensiones analizadas: implicaciones sociales de la ciencia $(\mathrm{S})$, adopción de actitudes científicas $(\mathrm{A})$ interés por la ciencia en el tiempo de ocio (L).

De manera globalizada, contemplando el valor medio alcanzado por cada dimensión, los datos parecen revelar la mayor tendencia actitudinal del alumnado en el siguiente orden: implicaciones sociales de la ciencia $(S)(\bar{X}=40,49 ; S x=5,26)$, adopción de actitudes científicas $(A)(\bar{X}=38,76 ; S x$ $=4,71)$ e interés por la ciencia en el tiempo de ocio $(L)(\bar{X}=35,91 ; S x=7,37)$. Estos datos se pueden relacionar con el reconocimiento de las implicaciones sociales de la ciencia en su entorno y contextos cotidianos, por parte del alumnado, en mayor medida que las otras dimensiones, apoyado por las manifestaciones continuas de su entorno escolar (profesorado, compañeros, centro) sobre la importancia de la investigación y la ciencia y quizá también en los medios de comunicación que la ciencia es importante. Estas percepciones en su progreso hacia la adopción de actitudes científicas en la vida personal se aminoran, puesto que requieren de una mayor interiorización que responda a que el/la estudiante haya asumido un comportamiento más coherente con una verdadera valoración o reconocimiento de la actividad científica pero interiorizada en diferente medida, en función del grado de compromiso e implicación que puedan conllevar. Así el interés por la ciencia en el tiempo de ocio, esfera que puede considerarse de mayor relajación personal y social, muestra los menores valores alcanzados, apuntando a la parcial incorporación que haya podido generarse en el "estar ciudadano" del estudiantado y en la proyección social con que ésta se ve reflejada.

De manera individual, por dimensiones, respecto a la implicación social de la ciencia (S), nuestro alumnado muestra una concienciación ciudadana importante sobre los temas científicos, si bien se detectan indecisiones en torno a aspectos de la financiación pública de la ciencia que pueden estar motivados por la escasa difusión y/o transparencia de las instituciones implicadas en darla a conocer a la sociedad civil, así como por la minoritaria asunción que científicos e investigadores ejercen, comunicando sus investigaciones y necesidades a la ciudadanía (FECYT, 2013, 2019).

En cuanto a la adopción de actitudes científicas (A), este es un predictor con menor desarrollo en la evolución de las percepciones y con más exigencias de interiorización. En concreto, en relación con esta dimensión, se encuentran ítems que reciben una destacada menor valoración (ítem 53 "No estoy dispuesto a cambiar mis ideas cuando la evidencia muestra que las ideas son malas" (43,1\%) $(\bar{X}=2,75 ; \mathrm{Sx}=1,27)$, o que muestran un amplio porcentaje de estudiantes indecisos (39\%) (ítem 60, "En experimentos científicos, informo de resultados inesperados y resultados esperados" $(\overline{\mathrm{X}}=3,46$; $\mathrm{Sx}=0,93)$. Indicios que nos hacen considerar que los estudiantes no han logrado integrar claramente la reflexión y la autocrítica ante la toma de decisiones, quizás por no tener asumida la ética científica en evidenciar los resultados que la experimentación muestra, sean los esperados o no, lo que induce a pensar que este aspecto no está muy incorporado en los programas educativos y, por tanto, no llega a trascender a los estudiantes. Comportamiento que es un exponente de una necesidad general mostrada por los ciudadanos en la sociedad actual y que precisa ser abordado en mayor profundidad en futuras formaciones, utilizando propuestas didácticas innovadoras (Girón, Lupión y Blanco, 2020) 
que promuevan una movilización de capacidades y competencias científicas vehiculando la asunción efectiva de los procesos comentados.

El interés por la ciencia en el tiempo de ocio (L) es la dimensión con menor valor medio global, alcanzando todos los ítems porcentajes moderados. En concreto ninguno de los ítems logra superar el $70 \%$, destacando el elevado porcentaje de indecisos (31\%), especialmente en el ítem 20 "Me gustaría recibir un libro de ciencias o un equipo científico como regalo" $(\bar{x}=3,40 ; S x=1,07)$, que evidencia el escaso apego en la percepción que se ha integrado al respecto, dado el escenario de consumo social que le retroalimenta (medios de comunicación, entorno cercano) que no le ofrecen la posibilidad de emplear su tiempo disfrutando con una ciencia más lúdica o de difusión de contenidos interesantes, sino mayoritariamente centrada en la promoción de productos y eventos de divulgación (Pérez-Rodríguez, González-Pedraz y Alonso-Berrocal, 2018).

Es de resaltar que en la comparativa entre ítems de todas las dimensiones, el de mayor porcentaje de valoración positiva (ítem 18 “Tengo curiosidad sobre el mundo en el que vivimos.") y menor (ítem 53 "No estoy dispuesto a cambiar mis ideas cuando la evidencia muestra que las ideas son malas") pertenecen a la misma dimensión sobre adopción de actitudes científicas, ocupa el segundo lugar en la media global entre dimensiones y que representa un escenario de comportamiento personal y social, con un nivel de compromiso intermedio entre ambas.

En cuanto a la comparativa por género, los resultados nos indican que las chicas parecen adoptar mayores actitudes científicas y tienen más interés por la ciencia en el tiempo de ocio que los chicos. Esto no se corresponde con la posterior formación científica superior, donde las chicas eligen estudiar carreras de ciencias en una proporción menor que lo hacen los chicos (FECYT, 2019; Vázquez y Manasero, 2009), lo que sugiere también una mejora en la formación u orientación que se da a las alumnas en los centros educativos.

La diferencia por niveles prevalece en favor en el progreso del mismo sugiriendo una incidencia efectiva de la incorporación de contenidos actitudinales en los valores del alumnado asociados al proceso formativo que experimenta en su educación científica al pasar de una educación obligatoria a otra postobligatoria, que ha elegido de forma opcional, con una orientación científica.

Entre las perspectivas de futuro, destacamos la importancia de contemplar estudios diagnósticos de las percepciones hacia la ciencia de nuestros estudiantes, para proyectar desde ellos, los objetivos de educación científica en la escuela, orientados a formar ciudadanos con alfabetización en CyT.

Mejorar las percepciones de nuestro alumnado es una oportunidad para encarar dicha orientación. Desde este interés, surge la necesidad de abordar enfoques de intervención educativa que potencien estas finalidades y desde la Didáctica de las Ciencias Experimentales, se aportan nuevos escenarios formativos y perspectivas de aprendizaje tendentes a dotar a nuestros estudiantes de conocimientos, capacidades y actitudes, para moverse como ciudadanos competentes en la sociedad, ante diferentes situaciones y contextos, mediante la utilización de innovadores enfoques de enseñanza y/o la utilización de estrategias metodológicas activas, fortalecedores no solo de conocimientos sino también de actitudes hacia la ciencia, como parte del currículo escolar.

Así, entre éstos podemos contemplar la utilización del enfoque de contextualización o el tratamiento STEM, que ofrecen propuestas movilizadoras de acercamiento desde las situaciones de vida diaria (Blanco, España y Rodríguez, 2012; Solbes et al., 2012), que pueden mostrar de forma más cercana el papel de la CyT, permitiéndole así al alumnado, entender mejor las contribuciones y utilidades que éstas aportan en la sociedad en que vivimos. Y también, la incorporación de las prácticas científicas de indagación, modelización y argumentación (Muñoz, Franco y Blanco, 2020), como tratamientos metodológicos que les permiten acercarse a la ciencia y analizar cómo identificar preguntas, recabar datos y analizarlos para alcanzar respuestas ante los temas sociocientíficos que la vida diaria nos presenta.

Por otro lado, la utilización de prácticas científicas de indagación e investigación (EC, 2014), o de argumentación (Jimenez-Aleixande y Erduran, 2009) son reconocidas como estrategias metodológicas adecuadas para mejorar la enseñanza de las ciencias y las matemáticas, estando muy 
constatada su implicación en el mayor clima motivacional del alumnado (Romine y Sadler, 2016). Relaciones a valorar en su implicación y en el fomento de conocimientos, capacidades y actitudes científicas (Vázquez y Manassero 2009; Aramendi, Arburua y Buján, 2018) en el alumnado, que desarrolla así un conocimiento en la acción, en un escenario de enseñanza donde interviene con una participación activa en su aprendizaje, interiorizando un mayor grado de compromiso e implicación $\mathrm{y}$ por tanto, gestando un reforzamiento en los procesos de reflexión, que contribuyan a la conformación de su identidad personal.

Por tanto, en aras a diseñar propuestas educativas futuras, sería relevante desarrollar en el futuro estudios longitudinales cuyo inicio tuviera lugar en $1^{\circ} \mathrm{ESO}$, permitiendo así disponer de una visión más amplia y dinámica de los procesos de desarrollo de las percepciones del alumnado hacia predictores sobre factores asociados a la función social de la ciencia, en aras a promover las vocaciones científicas que nuestra sociedad necesita.

\section{Referencias}

Aramendi P., Arburua R.M., Buján, K. (2018). El aprendizaje basado en la indagación en la enseñanza secundaria. Revista de Investigación Educativa, 36(1), 109-124.

Blanco, A., España, E. y Rodríguez, F. (2012). Contexto y enseñanza de la competencia científica. Alambique: Didáctica de las ciencias experimentales, 70, 9-18.

Caamaño, A. (2012). La investigación escolar es la actividad que mejor integra el aprendizaje de los diferentes procedimientos científicos. En E. Pedrinaci, A. Pro, A. Caamaño y P. Cañal (coords.), 11 ideas claves. El desarrollo de la competencia cientifica (pp. 127-146). Barcelona: Graó.

Cheung, D. (2009). Developing a Scale to Measure Students' Attitudes toward Chemistry Lessons.International Journal of Science Education, 31(16), 2185- 2203.

Couso, D. (2012). De la moda de "aprender indagando" a la indagación para modelizar: una reflexión crítica. Conferencia plenaria XXVI Encuentros de Didáctica de las Ciencias Experimentales. Huelva: APICE.

Declaración de Budapest (1999). Marco general de acción de la Declaración de Budapest. Recuperado de http://www.oei.org.co/cts/budapest.dec.htm

Delors, J. (Coord.) (1996). La educación encierra un tesoro. Informe a la UNESCO de la Comisión Internacional sobre la Educación para el siglo XXI. Madrid: Santillana. Ediciones UNESCO.

Consejo de Europa (2003). Conclusiones del Consejo de 5 de mayo de 2003 sobre los niveles de referencia del rendimiento medio europeo en educación y formación. Diario Oficial C 134 de 7.6.2003. Recuperado de https://op.europa.eu/es/publication-detail/-/publication/35b8e2a0a9c3-4b9b-8654-9a9c74ef2603

COSCE (Confederación de Sociedades Científicas de España) (2011). Informe ENCIENDE Enseñanza de las Ciencias en la Didáctica escolar por edades tempranas en España. Madrid: COSCE.

European Commission (2005). Special Eurobarometer 225 / Wave 63.1 Social values, Science and Technology. Brussels: autor. Recuperado de http://ec.europa.eu/public_opinion/archives/ebs/ebs_225_report_en.pdf

European Commision. (2015). Science education for responsible citizenship. Luxembourg. DOI: $10.2777 / 12626$.

Fernández-Sierra, E.M, Pérez-Pozo, A.J., Santiago-Ríos, F.J. y Navas-Parejo, M. (2019). Capítulo 21. La influencia de la gamificación en las actitudes del alumnado. Innovación Educativa en la Sociedad Digital, 729-739. Madrid: Dykinson.

Fundación Española para la Ciencia y la Tecnología (FECYT). (2013). Percepción social de la Ciencia y la Tecnología 2012. FECYT. 
Fundación Española para la Ciencia y la Tecnología (FECYT). (2019). Percepción social de la Ciencia y la Tecnología 2018. FECYT.

Fensham, P. (2009). Real world contexts in PISA science: Implications for contexts-basics science education. Journal of Research in Science Teaching, 46(8), 884-896.

Fraser, B. J. (1981). TOSRA: Test of science-related attitudes handbook. Hawthorn, Victoria: Australian Council for Educational Research.

Jiménez-Aleixandre M.P y Erduran, S. (2008). Argumentation in Science Education. En S. Erduran y M.P. Jiménez-Aleixandre (eds.). Argumentation in Science Education: Perspectives from Classroom-Based Research (pp. 3-27). Dordrecht, The Netherlands: Springer.

Girón-Gambero, J., Lupión-Cobos, T. y Blanco-López, A. (2020). Desempeño en competencias de estudiantes de $2^{\circ}$ de ESO en una propuesta ABP sobre la contaminación acústica. En T. SolaMartínez, M. García-Carmona, A. Fuentes-Cabrera, A.M. Rodríguez-García y J. LópezBelmonte, Innovación Educativa en la Sociedad Digital (pp. 106-117). Madrid: Dykinson.

Gormally, C., Brickman, P. y Lutz, M. (2012). Developing a Test of Scientific Literacy Skills (TOSLS): Measuring Undergraduates' Evaluation of Scientific Information and Arguments. The American Society for Cell Biology. CBE Life Science Education, 11(4), 364-377.

Klahr, D., Zimmerman, C. y Jirout, J. (2011). Educational interventions to advance children's scientific thinking. Science, 333(6045), 971-974.

Lozano, A. (2003). Factores personales, familiares y académicos que afectan al fracaso escolar en la Educación Secundaria. Revista Electrónica de Investigación Psicoeducativa y Psicopedagógica, 1, 43-66. DOI: 10.14204/ejrep

Lupión Cobos, T. y Blanco López, A. (2016). Reflexión sobre la práctica de profesorado de ciencias de secundaria en un programa formativo en torno a la competencia científica. Revista Electrónica Interuniversitaria de Formación del Profesorado, 19 (2), 195-206. DOI: 10.6018/reifop.19.2.253731

Lupión-Cobos, T., Franco-Mariscal, A.J. y Girón-Gambero, J. (2019). Predictores de vocación en Ciencia y Tecnología en jóvenes: Estudio de casos sobre percepciones de alumnado de secundaria y la influencia de participar en experiencias educativas innovadoras. Revista Eureka sobre Enseñanza y Divulgación de las Ciencias, 16(3), 3102. DOI: 10.25267/Rev_Eureka_ensen_divulg_cienc.2019.v16.i3.3102

Mayor Zaragoza, F. (2000). Un mundo nuevo. Barcelona: UNESCO. Círculo de Lectores.

Muñoz-Campos, V., Franco-Mariscal, A. J., y Blanco-López, A. (2020). Integración de prácticas científicas de argumentación, indagación y modelización en un contexto de la vida diaria. Valoraciones de estudiantes de secundaria. Revista Eureka sobre Enseñanza y Divulgación de las Ciencias, 17(3), 3201.DOI:10.25267/Rev_Eureka_ensen_divulg_cienc.2020.v17.i3.3201.

National Research Council (NCR) (2011). A framework for $K-\overline{1} 2$ science education: Practices, crosscutting concepts and core ideas. Washington, DC: National Research Council, Board on Science Education, Division of Behavioral and Social Sciences and Education.

Navarro, M., Förster, C., González, C. y González-Pose, P. (2016). Attitudes toward science: measurement and psychometric properties of the Test of Science-Related Attitudes for its use in Spanish-speaking classrooms. International Journal of Science Education, 38(9), 14591482. DOI: $10.1080 / 09500693.2016 .1195521$

Ryder, J., 2001. Identifying science understanding for functional scientific literacy. Studies in Science Education, 36,1-44.

Simarro, C. (2015). El fomento del interés por la educación científica entre los jóvenes: por un futuro mejor para los ciudadanos de mañana. En Obra Social “la Caixa”, FECYT y Everis, ¿Cómo podemos estimular una mente cientifica?, 5-11. Barcelona: Obra social de la Caixa y Fundación Española para la Ciencia y la Tecnología. 
Solbes, J., Furió, C., Domínguez, M. ${ }^{a}$ C., Fernández, J., Tarín, F. y Guisasola, J. (2012). What factors have an influence on a quality teaching practice in Sciences? Procedia-Social and Behavioral Sciences, 46, 4513-4517. DOI: 10.1016/j.sbspro.2012.06.287

Welch, A. (2010). Using the TOSRA to Assess High School Students' Attitudes toward Science after Competing In the FIRST Robotics Competition: An Exploratory Study. Eurasia Journal of Mathematics, Science \& Technology Education, 6(3), 187-197.

Vázquez A., Manassero M.A. (2008) El declive de las actitudes hacia la ciencia de los estudiantes: un indicador inquietante para la educación científica. Revista Eureka sobre Enseñanza y Divulgación de las Ciencias, 5(3), 274-292.

Vázquez, A., y Manassero, M. A. (2009). Patrones actitudinales de la vocación científica y tecnológica en chicas y chicos de secundaria. Revista Iberoamericana de Educación, 50(4), 115.

Unfried, A., Faber, M., Stanhope, D. y Wiebe, E. (2015). The development and validation of a measure of student attitudes toward science, technology, engineering, and math (S-STEM). Journal of Psychoeducational Assessment, 33(8), 1-18. DOI:10.1177/0734282915571160.

Zoller, U. (2012). Science Education for Global Sustainability: What is necessary for Teaching, Learning and Assessment Strategies? Journal of Chemical Education, 89, 297-300. DOI: $10.1021 / \mathrm{ed} 300047 \mathrm{v}$.

\section{CÓMO CITAR ESTE ARTÍCULO:}

Lupión Cobos, T. y Girón Gambero, J. R. (2020). Percepciones del alumnado de Educación Secundaria (15-17 años) hacia la función social de la ciencia. Didáctica de las Ciencias Experimentales y Sociales, 39,81-98. DOI: 10.7203/DCES.39.17766 